\title{
EDITORIAL
}

\section{Partial liquid ventilation}

\author{
S.J.C. Verbrugge, B. Lachmann
}

There is strong indication that modes of ventilation that lead to repeated closure and opening of surfactantdeficient alveoli can induce severe functional and histological lung damage [1]. New modes of ventilation are directed at prevention of lung injury due to mechanical ventilation by maintaining alveolar "gas volume" at end-expiration, by means of positive end-expiratory pressure ("gas PEEP"). Recent evidence suggests that such modes may prevent not only lung parenchymal injury but also proinflammatory mediator expression in the lung tissue [2], and bacterial translocation from the lung into the circulation [3]. The incidence of acute respiratory distress syndrome (ARDS) and multiple system organ failure (MSOF), which is still the leading cause of death in ARDS [4], may thus be reduced.

An alternative technique to maintain alveolar volume at end-expiration is by instilling perfluorocarbon (PFC) fluids into the lung, thereby providing "liquid PEEP" to the alveoli. Because PFCs dissolve high amounts of oxygen and carbon dioxide at normospheric pressures, gas exchange over the alveolar air-liquid interface is maintained when conventional mechanical gas ventilation is superimposed. This technique has become known as "partial liquid ventilation" (PLV) [5].

In this issue of the European Respiratory Journal, KAISERS et al. [6] show that mean short-term survival time in lung-lavaged pigs treated with PLV with low dose intratracheal PFC can be increased almost fourfold compared to a nontreated control group, when both groups are volume-control ventilated (tidal volume $=12$ $\mathrm{mL} \cdot \mathrm{kg}^{-1} ; \mathrm{PEEP}=5 \mathrm{cmH}_{2} \mathrm{O}$; inspiratory/expiratory $(\mathrm{I} / \mathrm{E})$ ratio $=1: 2$; fraction of inspired oxygen $\left.\left(F \mathrm{I}, \mathrm{O}_{2}\right)=1.0\right)$. The increased survival was attributed to a preserved oxygen delivery with PLV, which deteriorated in the nonPFC-treated control group. The authors also partially confirm previous experiences with PLV in animal models of acute lung injury (ALI), which have shown that:

1) Higher doses of PFC lead to higher levels of oxygenation [7]. This is suggested to result from dose-dependent recruitment of collapsed atelectatic alveoli by PFC fluid. Thereby, gas exchange also continues during the expiratory phase of the respiratory cycle.

2) Oxygenation deteriorates over time if no additional doses of PFC are applied [8]. This is attributed to evaporation of PFC, which will cause affected alveoli to collapse.

3) Lung mechanics and carbon dioxide elimination improve after an initial low dose of PFC and show no further improvements with subsequent higher doses of

Correspondence: B. Lachmann, Dept of Anesthesiology (Room Ee 2393), Erasmus University Rotterdam, Postbox 1738, 3000 DR Rotterdam, The Netherlands.
PFC [7]. This is attributed to the replacement of the alveolar air-liquid interface with a thin air-PFC interface. Evaporating PFC appears to cover the entire lung surface. As PFCs have a low constant surface tension (which is $12 \mathrm{mN} \cdot \mathrm{m}^{-1}$ in the case of FC 3280), pulmonary compliance is increased after a low-dose PFC and $\mathrm{CO}_{2}$ elimination is higher. No further improvement is seen after additional PFC dosing.

4) PLV does not impair any cardiovascular parameter; even in animals with a large anterior-posterior thoracic diameter. Mean pulmonary artery pressure decreases when PFC is applied, due to reversal of hypoxic pulmonary vasoconstriction [9].

5) PLV does prevent the progress of histologically assessed lung injury [10].

6) "Gas-PEEP" has to be applied during PLV to prevent bulk movement of PFC fluids from the alveoli into the airways and to prevent dangerously high airway pressures at the onset of inspiration [7].

7) PLV can be combined with other ventilatory support techniques in ALI, such as nitric oxide administration and high frequency oscillation $[11,12]$.

Although preliminary evaluations of phase II and III clinical studies with PLV have shown that PFCs can be safely applied to patients with ALI [13], these trials did not show the tremendous improvement of gas exchange that might have been anticipated from animal studies, nor could they demonstrate improvement in survival of patients treated with PLV compared to those treated with conventional mechanical ventilation only. There is a tendency towards an increased number of pneumothoraces in patients treated with PLV. How does one explain such findings?

\section{Possible side-effects of PLV}

There are some unclear aspects of PLV that may have adverse consequences for the lung. Firstly, although the alveolar air-liquid interface is eliminated when PFCs are instilled into the alveolus, a new interface with new surface tension is created at the air-PFC barrier. The law of Laplace: $P=2 \gamma / \mathrm{r}$, describes the relationship between the pressure to stabilize an alveolus $(P)$ and surface tension at the gas-liquid interface of an alveolus $(\gamma)$ in relation to the radius of the alveolus (r). In the normal "healthy" situation, surfactant present at the air-liquid interface in the lung has the unique property of lowering air-liquid surface tension in parallel with a decrease in alveolar radius (to almost $0 \mathrm{mN} \cdot \mathrm{m}^{-1}$ for low alveolar radii), thus keeping the ratio of $\gamma / \mathrm{r}$ of the alveolus constant, and guaranteeing expiratory alveolar stability at 
low pressures. PFC does not show this dynamic behaviour of surface tension. It maintains a constant air-PFC surface tension for any alveolar radius (which is $12 \mathrm{nN} \cdot \mathrm{m}^{-1}$ in the case of FC 3280).

The consequences are, firstly, that at end-expiration, the alveolar radius is small, whereas the surface tension is high $\left(12 \mathrm{mN} \cdot \mathrm{m}^{-1}\right)$. Alveoli will collapse if insufficient levels of "gas PEEP" are applied [14]. Therefore, "liquid PEEP" always needs to be accompanied by a high enough level of "gas PEEP" to prevent end-expiratory collapse of non-PFC-filled alveoli. As KAISERS et al. [6] point out in their discussion, insufficient PEEP levels may subject the alveolar wall to shear forces that may promote alveolar disruption and, successively, formation of pneumothoraces.

Secondly, concerning the level of "gas PEEP", it depends on the disease state of the individual alveolus whether or not the presence of a thin layer of PFC is beneficial. In those alveoli that are unable to reduce surface tension to $\leq 12 \mathrm{mN} \cdot \mathrm{m}^{-1}$ at minimal alveolar radii, the level of "gas PEEP" to keep alveoli open at endexpiration will be lower during PLV than the level of "gas PEEP" during conventional mechanical ventilation. However, in those alveoli that still have a surfactant system that is able to reduce surface tension to values below $12 \mathrm{mN} \cdot \mathrm{m}^{-1}$, there will be an interaction between PFC and the normal alveolar lining fluid, leading to levels of "gas PEEP" with PLV higher than those necessary to keep the alveoli open with "gas PEEP" only. Therefore, it may be important not to give PFC early in the disease process of ALI. For the same reason, the weaning period during the transition from PLV to normal gas breathing may be prolonged [14].

Another uncertainty with PLV is where the gas of the tidal breaths superimposed on the PFC is going. Suggesting the air would go to the non-fluid-filled parts of the lung, there could be a severe lung overinflation in those lung areas, due to the low surface tension of PFC, when conventional ventilation modes, such as volumecontrolled flow constant mechanical ventilation, are used. This may result in a high incidence of pneumothoraces with PLV. To prevent such complications, it is mandatory to combine fluid PEEP with pressure-controlled modes of ventilation, in which the pressure level in any alveolus can never exceed the pressure level set on the ventilator. This may prevent dangerous alveolar overdistension.

Finally, there are important in vitro changes to the cells of the immune system in the lung after exposure to PFCs. Exposure of alveolar macrophages to PFC decreases production of reactive oxygen metabolites upon chemical stimulation [15]; and neutrophils exposed to $\mathrm{PFC}$ produce less hydrogen peroxide $\left(\mathrm{H}_{2} \mathrm{O}_{2}\right)$ and show less chemotactic activity [16]. Whether such changes are beneficial in terms of reducing lung injury due to toxic oxygen radicals, or negative in terms of reduced host defence against intrapulmonary pathogens, needs further investigation.

\section{Future considerations}

Decreased oxygen delivery and increased oxygen consumption with increasing levels of lactate are correlat- ed to organ failure and mortality $[17,18]$. However, it is increasingly realized that the immune inflammatory system plays a major role in the pathophysiology of MSOF, and that the host's own endogenous inflammatory mediators, which may be released from the lung, contribute to organ failure and death in patients suffering from ARDS [19]. Recent preliminary data from animal studies have shown that PLV can reduce systemic release of proinflammatory mediators in an ALI model of acid aspiration [20]. However, the volume-controlled ventilator settings applied to the non-PFC-treated group in the study by KAISERS et al. [6] are not the ones currently propagated for ALI.

Improvements in oxygen delivery and survival time could probably also have been reached by counterbalancing the increased retractive forces in the alveoli by means of optimal ventilator settings with high end-expiratory gas volumes only and small pressure amplitudes [1]. Ventilator settings with "gas PEEP" alone have also been shown to reduce the amount of proinflammatory mediator expression in the surfactant-deficient lung due to mechanical ventilation [3]. This poses the question of whether "liquid PEEP" will have any additional value to optimal mechanical ventilation with "gas PEEP" alone in improving the survival of ARDS patient. Moreover, given the nondynamic surface tension behaviour of PFCs, restoring the physiological situation at the alveolar airliquid interphase in ARDS by means of exogenous surfactant therapy may prove to be superior to the application of PFCs.

Recent studies in surfactant-depleted lungs have shown that lung compliance can be improved to an even greater extent if intratracheal PFC administration is preceded by exogenous surfactant, which ensures a low interfacial surface tension at low alveolar volumes [21]. Future studies will have to demonstrate, firstly, whether PLV or surfactant, or a combination, is the best therapy to overcome respiratory failure and improve patient survival in ALI and, secondly, the impact of lung protective ventilatory strategies, such as high frequency oscillation and pressure-controlled inverse-ratio ventilation, in combination with "fluid PEEP" is superior to the use of these strategies with surfactant or "gas PEEP" alone. Only the ongoing phase III patient studies will show whether or not partial liquid ventilation has an impact on patient survival in acute lung injury.

\section{References}

1. Lachmann B. Open up the lung and keep the lung open. Intensive Care Med 1992; 18: 319-321.

2. Tremblay L, Valenza F, Ribeiro SP, Jingfang L, Slutsky AS. Injurious ventilatory strategies increase cytokines and c-fos m-RNA expression in an isolated rat lung model. J Clin Invest 1997; 99: 944-952.

3. Nahum A, Hoyt J, McKibben A, et al. Effect of mechanical ventilation strategy on $E$. coli pneumonia in dogs. Am Rev Respir Crit Care Med 1996; 153: A530.

4. Krafft P, Fridrich P, Pernerstorfer T, et al. The acute respiratory distress syndrome: definitions, severity and clinical outcome. Intensive Care Med 1996; 22: 519529.

5. Lachmann B, Verbrugge S. Liquid ventilation. Curr Opinion Crit Care 1996; 2: 60-66. 
6. Kaisers U, Max M, Kuhlen R, et al. Partial liquid ventilation with small volumes of FC 3280 increases survival time in experimental ARDS. Eur Respir J 1997; 10: 1955-1961.

7. Tütüncü AS, Faithfull S, Lachmann B. Intratracheal perfluorocarbon administration combined with mechanical ventilation in experimental respiratory distress syndrome: dose-dependent improvement of gas exchange. Crit Care Med 1993; 21: 962-969.

8. Tütüncü AS, Akpir K, Mulder P, Erdmann W, Lachmann B. Intratracheal perfluorocarbon administration as an aid in the ventilatory management of respiratory distress syndrome. Anesthesiology 1993; 79: 1083-1093.

9. Houmes RJM, Verbrugge SJC, Hendrik ER, Lachmann B. Hemodynamic effects of partial liquid ventilation with perfluorocarbon in acute lung injury. Intensive Care Med 1995; 21: 966-972.

10. Hirschl RB, Parent RB, Tooley R, et al. Liquid ventilation improves pulmonary function, gas exchange and lung injury in a model of respiratory failure. Ann Surg 1995; 21: 79-88.

11. Houmes RJM, Hartog A, Verbrugge SJC, Böhm SH, Lachmann B. Combining partial liquid ventilation with nitric oxide to improve gas exchange in acute lung injury. Intensive Care Med 1997; 23: 162-168.

12. Baden HP, Mellama JD, Bratton SL, O'Rourke PP, Jackson JG. High-frequency oscillation with partial liquid ventilation in a model of acute respiratory failure. Crit Care Med 1997; 25: 299-302.

13. Hirschl RB, Pranikoff T, Gauger P, Schreiner RJ, Dechert R, Bartlett RH. Liquid ventilation in adults, children and full-term neonates. Lancet 1995; 346: 1201-1202.

14. Salman NH, Fuhrman BP, Steinhorn DM, et al. Prolonged studies of perfluorocarbon-associated gas exchange and of the resumption of conventional mechanical ventilation. Crit Care Med 1995; 23: 919-924.

15. Smith TM, Steinhorn DM, Thusu K, Fuhrmann BP, Dandona P. A liquid perfluorochemical decreases the in vitro production of reactive oxygen species by alveolar macrophages. Crit Care Med 1995; 23: 1533-1539.

16. Rossman JE, Caty MG, Rich GA, Karamanoukian HL, Azizkhan RG. Neutrophil activation and chemotaxis after in vitro treatment with perfluorocarbon. $J$ Pediatr Surg 1996; 31: 1147-1151.

17. Kruse JA, Haupt MT, Puri VK, Carlsen RW. Lactate levels as predictors for the relationship between oxygen delivery and consumption in ARDS. Chest 1990; 98: 959-962.

18. Manikis P, Jankowski S, Zhang H, Kahn RM, Vincent JL. Correlation of serial blood lactate levels to organ failure and mortality after trauma. Am J Emerg Med 1995; 13: 619-622.

19. Demling RH. Adult respiratory distress syndrome: current concepts. New Horizons 1993; 1: 388-401.

20. Kawamae K, Pristine G, Chiumello D, Slutsky AS. Partial liquid ventilation decreases circulating TNF- $\alpha$ in an acid aspiration model. Am J Respir Crit Care Med 1997; 155: A87.

21. Tarczy-Hornoch P, Hildebrandt J, Mates EA, et al. Effects of exogenous surfactant on lung pressure-volume characteristics during liquid ventilation. J Appl Physiol 1996; 80: 1764-1771. 